\title{
Introducción al debate sobre la racionalidad de los impuestos
}

\author{
Introduction to the Debate about the \\ Rationality of Taxes
}

Alejandro Berrotarán*

Recepción y evaluación de propuesta: 18/02/2018

Aceptación: 01/07/2018

Recepción y aceptación final: 13/11/2020

Resumen: En "La racionalidad de los impuestos. Una tarea de reconciliación" Francisco Saffie aborda dos problemas centrales del derecho tributario contemporáneo: el déficit de justificación y la falta de cumplimiento de los deberes impositivos. A partir de un diagnóstico crítico de la situación actual, marcada por la concepción neoliberal, realiza una propuesta que se encamina a desmontar el andamiaje del paradigma clásico del derecho tributario para esbozar las bases de un paradigma alternativo superador. Eleonora Lozano Rodríguez, Eurico Marcos Diniz de Santi y Hernán Bouvier analizan la propuesta de Saffie y, a través de sus aportes y comentarios críticos, enriquecen la discusión sobre la temática. Finalmente, el autor principal da respuesta a cada uno de los cuestionamientos señalados y otorga un marco conceptual que clarifica su proyecto teórico.

Palabras claves: derecho tributario, legitimidad, elusión, evasión, solidaridad Abstract: In "The Rationality of Taxes. A Task of Reconciliation" Francisco Saffie addresses two central problems of contemporary tax law: the justification deficit and the lack of compliance with tax duties. Based on a critical

Abogado, Universidad Nacional de Córdoba, Córdoba, Argentina. Becario Doctoral del CONICET. Correo electrónico: alejandroberrotaran@derecho.unc.edu.ar 
Alejandro Berrotarán

diagnosis of the current situation, marked by the neoliberal conception, his proposal aims to dismantle the scaffolding of the classic paradigm of tax law in order to outline the basis of an alternative overcoming paradigm. Eleonora Lozano Rodríguez, Eurico Marcos Diniz de Santi and Hernán Bouvier analyze the proposal made by Saffie and, through their critical contributions and comments, enrich the discussion on the subject. Finally, the main author responds to the comments and provides a conceptual framework that clarifies his theoretical project.

Keywords: tax law, legitimacy, avoidance, evasion, solidarity

\section{Consideraciones iniciales}

El neoliberalismo ha destruido la posibilidad de que los arreglos institucionales de nuestras sociedades nos traten como iguales, afirma Francisco Saffie (2020). Es así, dice el profesor chileno, porque "promueve una distribución desigual del poder político y económico, que elimina los vínculos de solidaridad propios de la ciudadanía (Saffie, 2020)”. Francois Dubet a su vez señala que "la intensificación de las desigualdades procede de una crisis de las solidaridades, entendidas como el apego a los lazos sociales que nos llevan a desear la solidaridad de todos" (2015, p. 11). De esta manera, la falta de solidaridad y las desigualdades constituyen un tándem que se alimenta recíprocamente.

Diversas investigaciones empíricas, entre las que se destacan las últimas obras de Piketty (2014 y 2019), han evidenciado con claridad cómo desde hace décadas se está produciendo un fenómeno de escala mundial de concentración de riquezas en pocas manos. Ante esto, se han multiplicado las propuestas para, a través del derecho tributario, modificar los escandalosos niveles de desigualdad económica presentes ${ }^{1}$.

Saffie considera que es preciso ir más allá y presenta un proyecto que busca reivindicar la solidaridad como valor sobre el cual edificar el dere-

1 En Capital e Ideología, Piketty (2019) propone tributos altamente progresivos sobre la renta, las sucesiones y el patrimonio a la vez que una renta básica universal. Por su parte, Saffie (2012) defiende, entre otras medidas, un impuesto a la herencia para consolidar un sistema tributario más justo. 
cho tributario actual. Cree que para solucionar las desigualdades producto del neoliberalismo es necesario cambiar la forma en la que entendemos el sistema impositivo. Así, propone concebir a los impuestos ${ }^{2}$ no solo como instrumentos para recaudar y corregir variables económicas sino como una institución ligada a una práctica con un bien intrínseco.

Con estas ideas, el autor presenta en esta revista un diagnóstico sobre la situación actual marcada por lo que él denomina el paradigma clásico del derecho impositivo. Ante este panorama, propone una alternativa teórica para reconstruir y dar respuesta a los problemas que enfrentan nuestros sistemas tributarios.

Tres especialistas de diferentes nacionalidades latinoamericanas comentan el artículo de Saffie desde diversas perspectivas y realizando distintos aportes. En primer lugar, Eleonora Lozano Rodríguez de Colombia presenta sus dudas sobre algunas ideas vinculadas a la falta de cumplimiento de los deberes tributarios. Por su lado, Eurico Marcos Diniz de Santi de Brasil hace eje en tres conceptos que considera primordiales para solucionar los problemas diagnosticados: la transparencia, la simplicidad y la ciudadanía fiscal. Por último, Hernán Bouvier de Argentina cuestiona diferentes ideas defendidas por el autor principal: desde su caracterización del paradigma clásico hasta los puntos centrales de su propuesta teórica.

En esta introducción reconstruiré los ejes de "La racionalidad de los impuestos. Una tarea de reconciliación” de Saffie, presentaré algunas de las críticas que se le han realizado y concluiré con una reflexión sobre ciertos aportes que nos deja la discusión planteada.

\section{La legitimidad de los tributos}

En el artículo principal de este número de Discusiones, Francisco Saffie propone un abordaje de dos problemas que identifica como centrales en el derecho tributario: el de la legitimidad y el del cumplimiento de los deberes impositivos.

2 Para los fines de este artículo, y de la discusión en general que se presenta en este número, los términos "impuestos" y "tributos" serán tomados como sinónimos. 
Con respecto a la legitimidad, Saffie nos dice que el neoliberalismo ha menoscabado la función redistributiva de los impuestos y erosionado sus bases justificativas. La sensación de que los impuestos no cumplen su función redistributiva se debe, en parte, a la pérdida del sentido económico de los tributos ocasionada por el debilitamiento de los impuestos directos, el sustento del gasto público en impuestos indirectos y la disminución de las prestaciones estatales.

Saffie establece además que el déficit de legitimidad se debe a que se ha perdido el sentido político de los sistemas tributarios. El discurso neoliberal ha ocasionado una visión competitiva de las personas como unidades de capital humano. Así, se las concibe como "individuos aislados, luchando por sobrevivir, donde los intereses no pueden sino ser egoístas (Saffie, 2020)”. Este fenómeno provoca una destrucción de la solidaridad como bien público que sustenta a los sistemas impositivos más progresivos.

Las políticas neoliberales criticadas se asientan en un paradigma clásico del derecho tributario caracterizado por tres elementos: una justificación instrumental de los impuestos, una visión de las personas como seres racionales y egoístas, y una concepción de la propiedad privada como derecho natural. La crisis actual de legitimidad de los gravámenes impositivos evidencia las limitaciones de los presupuestos de este paradigma.

En su artículo, Bouvier cuestiona la falta de claridad conceptual cuando Saffie establece que el paradigma clásico "no da cuenta" de la racionalidad subyacente de los impuestos. Así, se pregunta: ¿El paradigma clásico no explica empíricamente la racionalidad de los impuestos? ¿ $\mathrm{O}$ el déficit explicativo es teórico? ¿O acaso lo denunciado es que esta visión no se encuentra normativamente justificada?

En su réplica, Saffie indica cómo el paradigma clásico es incapaz de explicar teóricamente la elusión impositiva y brinda una detallada respuesta para mostrar los déficits normativos de esta forma de ver a los tributos.

\section{El cumplimiento de los deberes tributario}

El segundo problema diagnosticado por Saffie es la falta de cumplimiento de los deberes tributarios a través de maniobras de elusión y evasión. Por 
un lado, la evasión ${ }^{3}$ se explica a partir de la pérdida de legitimidad ocasionada por el debilitamiento de los sentidos políticos y económicos de los sistemas impositivos. Por otro lado, la elusión ${ }^{4}$ se vincula con la teoría general del derecho tributario originada a partir del paradigma clásico. Esta teoría define al deber tributario como una relación jurídica en donde la obligación de pagar depende de la realización del hecho imponible. En tanto no se verifiquen los factores y las condiciones operativas que definen a este hecho, se permite un amplio espacio para acciones exentas del deber de contribuir. Esta estructura legal rígida establece un límite a una interpretación sustantiva o finalista de las normas que haría que conductas elusivas, que no configuran el hecho imponible, se vean obligadas a tributar.

Lozano se muestra escéptica ante esta descripción. En base a investigaciones propias, la autora señala que existen diversas motivaciones que llevan a quien contribuye a incumplir sus obligaciones tributarias. En este sentido, si la base motivacional para el comportamiento elusivo o evasivo es múltiple, entonces una propuesta teórica como la de Saffie, focalizada en la solidaridad, no es suficiente para solucionar el problema de falta de cumplimiento de las normas tributarias.

Por su parte, Santi disiente con las prioridades establecidas por Saffie y considera que existen ciertos desafíos que preceden al abordaje realizado. Así, a partir de la situación tributaria de Brasil, establece una propuesta que gira en torno a tres ideas centrales: la transparencia, la simplicidad y la ciudadanía fiscal.

Con respecto a la transparencia, Santi señala que uno de los ejes centrales del problema de la falta de legitimidad y cumplimiento de los deberes tributarios es que quienes contribuyen impositivamente no saben cómo, cuándo, cuánto y por qué contribuyen. A su vez, considera que es necesario simplificar la legislación. El caso brasileño presentado por el autor es un ejemplo paradigmático de la complejidad tributaria denunciada, pero ade-

3 Entendida como el empleo de medios abiertamente ilegales para evitar o reducir el pago de tributos.

4 “(S)egún la definición canónica, la elusión tributaria supone que, incluso allí donde existen reglas que determinan deberes legales, los individuos actuarían legalmente si evitan esos deberes cuando su comportamiento no está descrito de manera literal en la norma que establece el impuesto (Saffie, 2016, p. 440)". 
más parece caracterizar una situación generalizada. En este sentido, Dubet, haciendo referencia a Francia, afirma que "el sistema sobre el cual se basa la solidaridad se ha vuelto tan complejo que, finalmente, sus fundamentos simbólicos resultan metódicamente destruidos" (2015, p. 91).

Finalmente, con ideas cercanas a la propuesta republicana de una ciudadanía activa como "eterna vigilante" de las actividades públicas (Pettit, 1997), Santi propone el desarrollo de una ciudadanía fiscal que permita que, una vez superadas la oscuridad y complejidad del sistema impositivo, tenga la posibilidad de controlar y participar en la construcción de las políticas tributarias.

Saffie responde a Lozano que la tarea que él se propone no es tanto de ética tributaria sino de justificación moral de los tributos. Con respecto a lo comentado por Santi, el autor principal dice que sus preocupaciones difieren. Mientras que para el especialista brasilero la preocupación es cómo lograr una comprensión y acatamiento a los deberes impositivos, para él el desafío es ofrecer un marco filosófico alternativo para justificar los tributos. Para avanzar en estas respuestas, veamos algunos de los ejes de su propuesta.

\section{Propuesta teórica alternativa}

A partir de un marco teórico hegeliano, Saffie entiende que se deben abandonar los intentos de justificación de la tributación a partir de una lógica instrumental. Por el contrario, propone abordar cuál es el bien interno propio de esta práctica que la justifica.

Según las ideas del autor, "las reglas que dan forma (a las) instituciones tienen un contenido ético que las excede" (Saffie, 2016, p. 418). De esta manera, propone reinterpretar las instituciones desde una concepción relacional en donde éstas tienen una justificación intrínseca. En este sentido aclara, "(d)e no tener en cuenta este contenido ético las instituciones pueden dar lugar a comportamientos que solo en apariencia quedan fuera del contenido de las normas y niegan sus propósitos" (Saffie, 2016, p. 425), como los son las maniobras elusorias.

El bien que el autor identifica como intrínseco en la práctica de los impuestos es el reconocimiento recíproco y el desarrollo de virtudes ligadas a la 
dependencia mutua. Estos valores, que justifican al sistema tributario, responden a las demandas de fraternidad que supone la igualdad que nos debemos.

Finalmente, Saffie, se aleja de la premisa del paradigma clásico que indica que existe una propiedad privada natural o anterior a la existencia de los tributos. A partir de Murphy y Nagel, considera que la propiedad se justifica por el valor que representa para las personas, depende de la concepción de justicia adoptada y es conceptualmente inseparable de los impuestos. Según esta caracterización, y siguiendo nuevamente a Hegel, sostiene que la propiedad privada posibilita un espacio para la libertad al permitir la expresión de la voluntad personal. El límite para el reconocimiento de la expresión de la subjetividad individual se encuentra en el espacio para el desarrollo de la subjetividad de las demás personas. En este sentido, propiedad privada y sistema tributario serán los mecanismos para asegurar la libertad de todas las personas y la posibilidad para su desarrollo como agentes libres.

Bouvier presenta dos críticas a la propuesta teórica planteada. Por un lado, cuestiona la forma en la que se pretende solucionar el problema de la elusión a través de una interpretación finalista de las normas tributarias. Ya que si la propuesta de Saffie busca que acciones consideradas elusivas en el sistema de reglas caigan bajo el rótulo de evasivas con un diseño encaminado a una interpretación sustantiva, esto implicaría una forma de desprecio ante quien contribuye. Esto en la medida en que la conducta de quien obra de manera preventiva y se adapta a lo que el texto legal establece, podría ser considerada evasiva bajo una interpretación sustantiva de la norma.

Por otro lado, Bouvier muestra algunas dificultades del aparato conceptual incorporado por Saffie a partir de las tesis de MacIntyre y Honneth. Así, cuestiona la caracterización de la noción de práctica en contraste con la de institución y se muestra escéptico ante el potencial de la idea de bien interno como instrumento argumentativo para criticar al paradigma clásico y sustentar una visión alternativa del sistema tributario. A su vez, dice que, aun suponiendo la necesidad del reconocimiento recíproco para el desarrollo autónomo, no se explicita en dónde se fundamenta la expectativa normativa para que la esfera respectiva a los tributos forme parte de la práctica de reconocimiento. 
Alejandro Berrotarán

\section{Reflexiones finales}

En su texto final, Saffie elabora una réplica sofisticada y detallada en la que responde a los múltiples cuestionamientos que se le realizan. En esta tarea argumentativa en la que se embarca el autor, dota de robustez teórica y claridad conceptual a los múltiples puntos abordados en su artículo central. Así, sus respuestas enriquecen de manera significativa sus ideas originarias y otorgan un panorama teórico general capaz de consolidar la alternativa teórica propuesta.

¿Son sus respuestas capaces de enfrentar los cuestionamientos presentados por quienes participan de esta discusión? ¿Ofrece una solución plausible a los problemas de falta de legitimidad y de cumplimiento de los deberes impositivos en un contexto neoliberal? ¿Propone un aparato conceptual que permita sustentar una visión alternativa al paradigma clásico del derecho tributario? Quien lea la discusión presentada será quien responda estas preguntas.

Sin perjuicio de lo anterior, la discusión planteada ha logrado importantes resultados. En primer lugar, ha sido capaz de conectar las perspectivas teóricas de cuatro especialistas de Latinoamérica fortaleciendo los diálogos académicos en esta región del mundo. A su vez, los desafíos presentados por quienes discutieron el texto original han consolidado una propuesta alternativa sobre la forma de explicar y justificar el derecho tributario que, sin dudas, contribuirá al desarrollo académico sobre la temática. Por último, el abordaje realizado permite enriquecer el debate público y avanzar hacia arreglos institucionales más justos en un tema de indudable actualidad y relevancia en nuestras sociedades.

\section{Bibliografía}

Dubet, F. (2015). ¿Por qué preferimos la desigualdad? (aunque digamos lo contrario). Buenos Aires: Siglo Veintiuno Editores.

Pettit, P. (1997). Republicanism. A Theory of Freedom and Government. Oxford: Oxford University Press. 
Piketty, T. (2014). El capital en el siglo XXI. México D.F.: Fondo de cultura económica.

Piketty, T. (2019). Capital e ideología. Ciudad de Buenos Aires: Paidós.

Saffie, F. (2012). El impuesto a las herencias como una institución de justicia. Estudios Públicos, 126, pp. 123-161.

Saffie, F. (2016). Una crítica a las concepciones dualista y monista de la justicia distributiva igualitaria. En Gallego J. (coord.) Igualitarismo: una discusión necesaria, (pp. 415 a 446). Santiago de Chile: Centro de Estudios Públicos.

Saffie, F. (2020). Los impuestos no son armas: repensando los vínculos sociales tras el 18/O. Santiago de Chile: Centro de Investigaciones en Periodismo. Recuperado de: https://www.ciperchile.cl/2019/10/26/ los-impuestos-no-son-armas-repensando-los-vinculos-sociales-trasel-18-o/ 